\title{
ON A THEOREM OF HIGHER RECIPROCITY*
}

\section{BY ALBERT WHITEMAN $\dagger$}

1. Introduction. Let $\mathfrak{D}$ denote the totality of polynomials in an indeterminate $x$, with coefficients in a fixed Galois field $G F\left(p^{\pi}\right)$ of order $p^{\pi}$. Let $P$ be a primary irreducible polynomial in $\mathfrak{D}$; then, if $A$ is any polynomial in $\mathfrak{D}$ not divisible by $P$, we define $\{A \mid P\}$ as that element in $G F\left(p^{\pi}\right)$ for which

$$
\left\{\frac{A}{P}\right\} \equiv A^{\left(p^{\pi \nu}-1\right) /\left(p^{\pi}-1\right)} \quad(\bmod P),
$$

where $\nu$ is the degree of $P$.

We have then the following theorem of reciprocity due to $H$. Kuhne $\ddagger$ and rediscovered by Schmidt $\S$ and Carlitz.\|

If $P$ and $Q$ are primary irreducible polynomials in $\mathfrak{D}$ of degree $\nu$ and $\rho$ respectively, then

$$
\left\{\frac{P}{Q}\right\}=(-1)^{\rho \nu}\left\{\frac{Q}{P}\right\} .
$$

If $M=P_{1}^{a_{1}} \cdots P_{k}^{a_{k}}$ and $(A, M)=1$ we use the definition,

$$
\left\{\frac{A}{M}\right\}=\left\{\frac{A}{P_{1}}\right\}^{a_{1}} \ldots\left\{\frac{A}{P_{k}}\right\}^{a_{k}} .
$$

The purpose of this note is to give a simple new proof of the following theorem:

* Presented to the Society, February 20, 1937.

$\dagger$ Harrison Scholar in Mathematics, University of Pennsylvania.

$\ddagger \mathrm{H}$. Kuhne, Eine Wechselbeziehung zwischen Funktionen mehrerer Unbestimmter die zu Reziprozitätsgesetzen filhrt, Journal für die reine und angewandte Mathematik, vol. 124 (1901-02), pp. 121-133.

$\S \mathrm{F}$. K. Schmidt, Zur Zahlenthearie in Körpern von der Charakteristik p, Sitzungsberichte der Physikalish-medizinischen Societät zu Erlangen, vol. 58-59 (1928), pp. 159-172.

|| L. Carlitz, The arithmetic of polynomials in a Galois field, American Journal of Mathematics, vol. 54 (1932), pp. 39-50. 
If $M$ and $N$ are primary relatively prime polynomials in $\mathfrak{D}$ of degree $m$ and $n$ respectively, then

$$
\left\{\frac{M}{N}\right\}=(-1)^{m n}\left\{\frac{N}{M}\right\} .
$$

This generalized form of Kuhne's theorem is, of course, not new. The novelty of our method consists in proving the case $M, N$ directly (rather than $P, Q$ ) by making use of the generalized analog of Gauss's lemma* proved in $\$ 2$.

2. Generalization of the Analog of Gauss's Lemma. We shall employ the following notation. If

$$
F=a_{0} x^{\nu}+a_{1} x^{\nu-1}+\cdots+a_{\nu}, \quad a_{0} \neq 0,
$$

is a polynomial in $\mathfrak{D}$, then

$$
\operatorname{sgn} F=a_{0}, \quad \operatorname{deg} F=\nu ;
$$

for sgn $F=1, F$ is said to be primary. Let $R(A / B)$ denote the remainder in the division of $A$ by $B$. Then the generalization in question is furnished by the following lemma.

Lemma. Let $A$ and $M$ be in $\mathfrak{D}, M$ primary and relatively prime to $A$; then

$$
\left\{\frac{A}{M}\right\}=\prod_{\operatorname{deg} H<m} \operatorname{sgn} R\left(\frac{H A}{M}\right),
$$

the product extending over all primary $H$ of degree less than the degree of $M$.

We shall now give a proof of this lemma along lines suggested by Schering's $†$ proof in the numerical case.

3. Proof of the Lemma. Following Dedekind, $\ddagger$ we define $\phi(M)$ to be the number of polynomials in a reduced residue system, $\bmod M$; the number of primary polynomials prime to $M$

* L. Carlitz, loc. cit., p. 46.

$\dagger$ E. Schering, Zur Theorie der quadratischen Reste, Acta Mathematica, vol. 1 (1882), pp. 153-170; see also P. Bachmann, Die Elemente der Zahlentheorie, 1892, pp. 144-148.

$\ddagger$ R. Dedekind, Abriss einer Theorie der höheren Congruenzen in Bezug auf einer reellen Primzahl-Modulus, Journal für die reine und angewandte Mathematik, vol. 54 (1857), pp. 1-26. 
and of degree less than $m$ is then evidently $\phi(M) /\left(p^{\pi}-1\right)$. Hence, just as in the numerical case, it is very easy to show that the number of primary polynomials $H$ of degree less than $m$ such that $(H, M)=D$ is $\phi(M / D) /\left(p^{\pi}-1\right)$.

Put $H=H_{1} D, M=M_{1} D$. Then the congruence

$$
H A \equiv H^{\prime} \operatorname{sgn} R\left(\frac{H A}{M}\right) \quad(\bmod M), \operatorname{deg} H^{\prime}<m, \operatorname{sgn} H^{\prime}=1,
$$

becomes

$$
H_{1} A \equiv H_{1}^{\prime} \operatorname{sgn} R\left(\frac{H A}{M}\right) \quad\left(\bmod M_{1}\right) .
$$

Evidently the polynomials $H_{1}$ are the polynomials $H_{1}^{\prime}$ in some order. Therefore, if we multiply all congruences of the type (1) together and divide each member of the resulting congruence by the product of the $H_{1}$ (which is prime to $M_{1}$ ), we have

$$
A^{\phi\left(M_{1}\right) /\left(p^{\pi}-1\right)} \equiv \prod_{(H, M)=D} \operatorname{sgn} R\left(\frac{H A}{M}\right) \quad\left(\bmod M_{1}\right) .
$$

For $M_{1}=P$, a primary irreducible polynomial of degree $\nu$, the last congruence becomes

$$
A^{\left(p^{\pi \nu}-1\right) /\left(p^{\pi}-1\right)} \equiv\left\{\frac{A}{P}\right\} \quad(\bmod P) .
$$

Writing this congruence in the form

$$
A^{\left(p^{\pi \nu}-1\right) /\left(p^{\pi}-1\right)}=\left\{\frac{A}{P}\right\}+F P,
$$

and raising both members to the $p^{\pi(k-1)}$ th power, we can readily show that

$$
A^{p^{\pi(k-1) \nu}\left(p^{\pi \nu}-1\right) /\left(p^{\pi}-1\right)}=\left\{\frac{A}{P}\right\}+F^{\prime} P^{p^{\pi(k-1) \nu}} .
$$

But it is well known that

$$
\phi\left(P^{k}\right)=p^{\pi(k-1) \nu}\left(p^{\pi \nu}-1\right) .
$$

\section{Hence}




$$
A^{\phi\left(P^{k}\right) /\left(p^{\pi}-1\right)} \equiv\left\{\frac{A}{P}\right\} \quad\left(\bmod P^{k}\right) .
$$

Finally, for $M_{1}=P_{1}^{b_{1}} \cdots P_{k^{b_{k}}}, 0 \leqq b_{i} \leqq a_{i}, k>1$, deg $P_{i}=\nu_{i}$, we have

$$
\frac{\phi\left(M_{1}\right)}{p^{\pi}-1}=\frac{1}{p^{\pi}-1} \prod_{i=1}^{k} p^{\left(b_{i}-1\right) \pi \nu_{i}}\left(p^{\pi \nu_{i}}-1\right) .
$$

Hence, since

it follows that

$$
A^{p^{\pi \nu i}} \equiv 1\left(\bmod P_{i}\right)
$$

$$
A^{\phi\left(M_{1}\right) /(p \pi-1)} \equiv 1\left(\bmod M_{1}\right),
$$

where, as already stated, $M_{1}$ is the product of at least two distinct irreducible polynomials.

Combining the results of $(2), \cdots,(5)$ we now see that

$$
\prod_{(H, M)=D} \operatorname{sgn} R\left(\frac{H A}{M}\right)
$$

has the value 1 unless $M_{1}=M / D$ is irreducible or the power of an irreducible polynomial. On the other hand, for $M_{1}=P_{i}{ }^{b}$ $\left(b=1, \cdots, a_{i}\right),(6)$ has the value $\left\{A \mid P_{i}\right\}$. Consequently

$$
\begin{aligned}
\prod_{D \mid M} \prod_{(H, M)=D} \operatorname{sgn} R\left(\frac{H A}{M}\right) & =\prod_{\operatorname{deg} H<m} \operatorname{sgn} R\left(\frac{H A}{M}\right) \\
& =\left\{\frac{A}{P_{1}}\right\}^{a_{1}} \cdots\left\{\frac{A}{P_{k}}\right\}^{a_{k}}
\end{aligned}
$$

from which the Lemma follows at once.

4. Proof of the Theorem. Let $A, N$ denote primary polynomials of degrees $a, n$ respectively; let $(A, N)=1, a \geqq n$. Consider the congruence

$$
A \equiv \mathbb{R}(A / N) \quad(\bmod N), \quad \operatorname{deg} R(A / N)<n .
$$

Evidently there exists a primary $H$ (say $H_{0}$ ) of degree $a-n$ such that

$$
A=R(A / N)+H_{0} N .
$$

But this equation may be written in the form 


$$
H_{0} N \equiv-\mathbb{R}(A / N) \quad(\bmod A) .
$$

Let $E$ be any polynomial (not necessarily primary) of degree less than $a-n$. Then we may write

(8) $\left(H_{0}+E\right) N \equiv E N-\mathbb{R}(A / N)(\bmod A)$,

where

$$
0<\operatorname{deg}(E N-R(A / N))<a
$$

$$
\operatorname{sgn}(E N-R(A / N))=\operatorname{sgn} E N=\operatorname{sgn} E .
$$

Furthermore, we have the obvious identity

$$
\prod_{\operatorname{deg} H=a-n} H N=H_{0} N \prod_{\operatorname{deg} E<a-n}\left(H_{0}+E\right) N, \quad E \neq 0 .
$$

Therefore, by equations $(7), \cdots,(10)$,

$$
\begin{aligned}
\prod_{\operatorname{deg} H=a-n} \operatorname{sgn} & R\left(\frac{H N}{A}\right) \\
= & \operatorname{sgn} R\left(\frac{H_{0} N}{A}\right) \prod_{\operatorname{deg} E<a-n} \operatorname{sgn} R\left(\frac{\left(H_{0}+E\right) N}{A}\right) \\
& =-\operatorname{sgn} R\left(\frac{A}{N}\right) \prod_{\operatorname{deg} E<a-n} \operatorname{sgn} E .
\end{aligned}
$$

Now, by the generalization of Wilson's theorem for a Galois field,

$$
\prod_{b} b=-1, \quad b \text { in } G F\left(p^{\pi}\right),
$$

from which it follows at once that

$$
\prod_{\operatorname{deg} E<a-n} \operatorname{sgn} E=(-1)^{a-n} .
$$

Hence (11) becomes

$$
\prod_{\operatorname{deg} H=a-n} \operatorname{sgn} \mathcal{R}\left(\frac{H N}{A}\right)=(-1)^{a-n+1} \operatorname{sgn} \mathcal{R}\left(\frac{A}{N}\right) .
$$

Since

$$
R\left(\frac{H N}{A}\right)=-R\left(\frac{A}{H N}\right), \operatorname{deg} H N=\operatorname{deg} A,
$$


(12) may also be written in the form

$$
\prod_{\operatorname{deg} H=a-n} \operatorname{sgn} R\left(\frac{A}{H N}\right)=(-1)^{a-n} \operatorname{sgn} R\left(\frac{A}{N}\right) .
$$

Let us now assume, as we may without any loss of generality, that $m \geqq n$. In (12) replace $A$ by $K M$, where $K$ is any primary polynomial of degree $k(k<n)$. Then we have

$$
\prod_{\operatorname{deg} H=k+m-n} \operatorname{sgn} R\left(\frac{H N}{K M}\right)=(-1)^{k+m-n+1} \operatorname{sgn} R\left(\frac{K M}{N}\right) .
$$

Now let $K$ run through all the $p^{\pi k}$ primary polynomials of degree $k$; we get

$$
\prod_{\substack{\operatorname{deg} H=k+m-n \\ \operatorname{deg} K=k}} \operatorname{sgn} R\left(\frac{H N}{K M}\right)=(-1)^{k+m-n+1} \prod_{\operatorname{deg} K=k} \operatorname{sgn} R\left(\frac{K M}{N}\right) .
$$

In a similar manner we may obtain from (13),

$$
\prod_{\substack{\operatorname{deg} H=k+m-n \\ \operatorname{deg} K=k}} \operatorname{sgn} R\left(\frac{H N}{K M}\right)=(-1)^{k} \prod_{\operatorname{deg} H=k+m-n} \operatorname{sgn} \mathcal{R}\left(\frac{H N}{M}\right)
$$

Comparing (14) and (15), we obtain

$$
\prod_{\operatorname{deg} K=k} \operatorname{sgn} R\left(\frac{K M}{N}\right)=(-1)^{m+n-1} \prod_{\operatorname{deg} H=k+m-n} \operatorname{sgn} R\left(\frac{H N}{M}\right) .
$$

Therefore

$$
\prod_{\operatorname{deg} K<n} \operatorname{sgn} R\left(\frac{K M}{N}\right)=(-1)^{m n+n^{2}-n} \prod_{m-n \leqq \operatorname{deg} H<m} \operatorname{sgn} R\left(\frac{H N}{M}\right) .
$$

When we note that

$$
\prod_{\operatorname{deg} H<m-n} \operatorname{sgn} R\left(\frac{H N}{M}\right)=1
$$

the theorem follows at once.

University of Pennsylvania 